\title{
In vitro and in vivo investigations toward near-field microwave-based detection of melanoma
}

\author{
David Dubuc, Katia Grenier \\ LAAS -CNRS, Université de Toulouse, Toulouse, France \\ dubuc@1aas.fr ; grenier@1aas.fr
}

\author{
Florent Morfoisse, Barbara Susini-Garmy \\ INSERM UMR 1048, CHU Rangueil, 1, av Jean Poulhès, \\ 31432 Toulouse Cedex 4, France \\ barbara.garmy-susini@inserm.fr
}

\begin{abstract}
To go toward non-invasive melanoma analysis without the requirement of biopsy, in vitro and in vivo investigations have been performed with near-field microwave sensing techniques. Preliminary dielectric study has been carried out on different types of skin crushes: normal skin and skin with a subcutaneous B16 melanoma tumor. Extracted dielectric properties permits to clearly discriminate the two samples. Based on these results, in vivo investigations -have been performed on living mice, leading to a possible microwave-based detection of melanoma, which should now be confirmed with statistical measurements. The dielectric results are in agreement with the observation through immune-analysis of increased vascular density in the presence of a tumor, i.e. water content rise.
\end{abstract}

Keywords-microwave; biosensor; melanoma; skin cancer; permittivity

\section{INTRODUCTION}

Early and non-invasive detection of malignant melanoma constitutes an important challenge for this low vital prognostic pathology. This type of skin cancer is indeed particularly metastatic and its early diagnosis is proven to drastically increase the chance of recovery and survival.

Nowadays, malignant melanoma diagnosis is performed by trained dermatologists in complementarity to optically-based microscopic equipment and followed by biopsies. On the other side, the discrimination between healthy and malignant organs and tissues has already been demonstrated with electrical measurements on a large frequency range. In the microwave range, dielectric spectroscopy is notably under development as an alternative imaging solution for breast or liver cancers in particular [1]-[3], reaching nowadays the clinical trials stage. Radiofrequency waves present indeed the attractive features of being non-ionizing and non-invasive. Therefore, microwave and millimeterwave reflectometry has also been reported for skin analysis [4]-[7] with different topologies, technologies and frequency ranges.

In the $\mathrm{kHz}-\mathrm{MHz}$ range, it even led to the development of a commercial equipment, NeviSense from Scibase, based on electrical impedance spectroscopy up to $2.5 \mathrm{MHz}$ (http://scibase.se/en/the-nevisense-product/) [8]. In this case, electrode tip was developed as minimally invasive, as it integrates very tiny spikes on the surface.
In this paper, we focused on developing microwave-based near-field sensing in the prospective of providing a low cost, non-invasive and rapid melanoma detection instrument; which would enable an early diagnostic without any requirement of biopsy.

Toward this purpose, a preliminary in vitro study has been conducted with skin samples of mice, with and without inoculated melanoma tumor cells, in order to demonstrate the possible discrimination of both skin types using skin crushes in a laboratory test setup, which enables the extraction of dielectric properties. Next section is dedicated to in vivo investigations on living mice based on a developed microstrip sensor realized with a low-cost PCB technology and associated to a portable microwave test setup for non-invasive melanoma detection.

\section{II. -IN VItRo MicrowaVE MEASUREMENTS OF SKIN CRUSTS}

\section{A. Sample preparation for in vitro study}

Mice have been subcutaneously inoculated with B16 melanoma tumor cells. After incubation, a tumor has been formed, as shown in Fig. 1 on an asleep mouse, which has been shaved for better visualization of the tumor under skin.

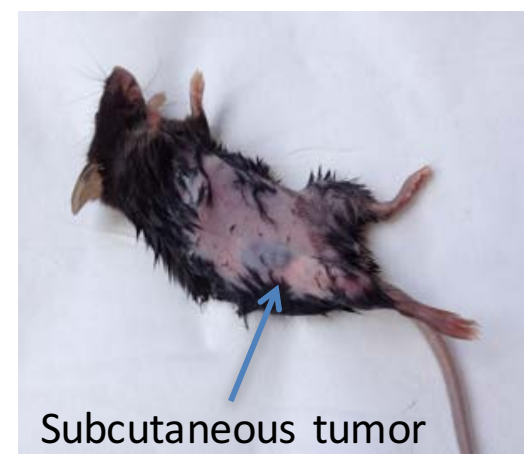

Fig. 1. Visualization of the subcutaneous tumor on a mouse, which hair has been removed in the tumor zone for optical observation and skin sampling

The mouse has then been sacrificed. Normal skin and skin with tumor have then been collected, crushed and the same 
quantity for both samples has been suspended in the same biological buffer.

\section{B. Microwave sensing of skin samples}

To perform the dielectric characterization of the two fluidic skin samples, a laboratory microwave test setup developed in the team has been used. A Vector Network Analyser (VNA) is connected to a miniature microwave sensor placed on a probestation. The fluid ( 2 microliters only) is injected into a microfluidic channel on top of a coplanar waveguide, which acts as microwave near-field sensor, as presented in Fig. 2. Broadband dielectric characterization of fluids may then be performed from $40 \mathrm{MHz}$ to $40 \mathrm{GHz}$. Details about the test setup and permittivity extraction from $\mathrm{S}$ parameters measurements may be found in [9]-[11].

A/

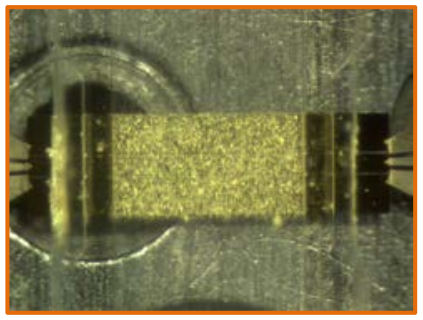

B/

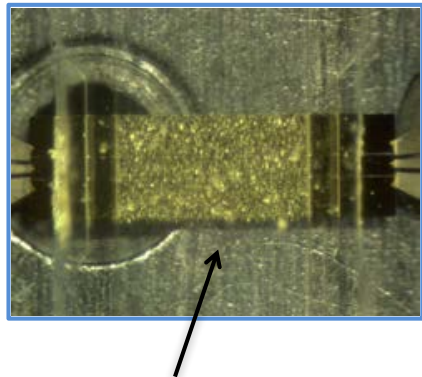

Crushed extract in the microfluidic channel of the sensor

Fig. 2. Microwave sensor loaded with crushed extracts of normal skin (A) and skin with melanoma (B).

Fig. 2 presents the coplanar sensor loaded by the two different samples. Real and imaginary parts of fluids permittivity may then be extracted versus frequency. To enable a precise comparison of the two samples, the permittivity of the biological buffer is subtracted from the permittivity of the "skin" fluid for both real and imaginary parts and leads to permittivity contrasts.

The dielectric contrasts on the real part of permittivity for both healthy (blue) and tumorous (orange) skin fluidic samples are then presented in Fig. 3. The host medium (biological buffer) corresponds to the zero line in abscissa. Dielectric measurements have been replicated in the microsensor.

The results indicate a clear discrimination between both samples. Based on this first validation, in vivo microwavebased measurements have been conducted.

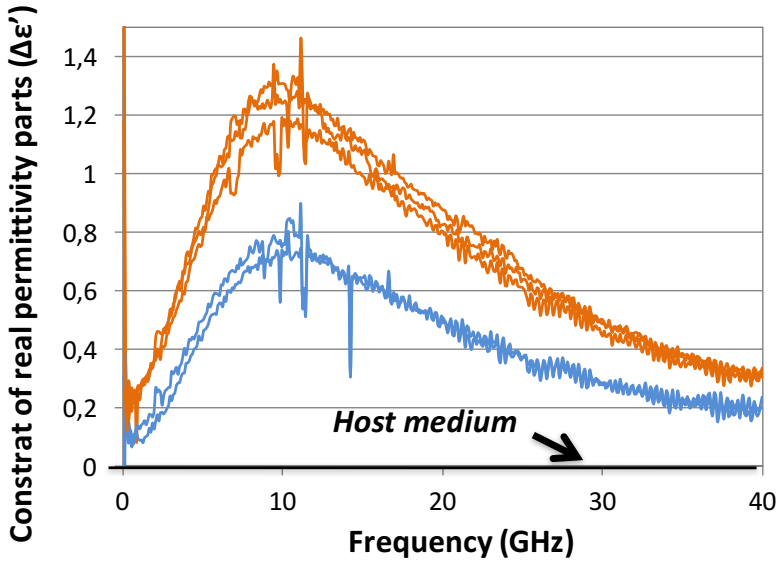

Fig. 3. Dielectric contrasts on the real part of permittivity for the control (blue) and tumorous (orange) skin fluidic samples.

\section{IN VIVO STUDY}

\section{A. Biological observation of tissues cross sections}

In parallel to in vivo microwave sensing, immunocharacterizations of mice skin tissues have been performed. The corresponding results on cryosections are indicated in Fig. 4.

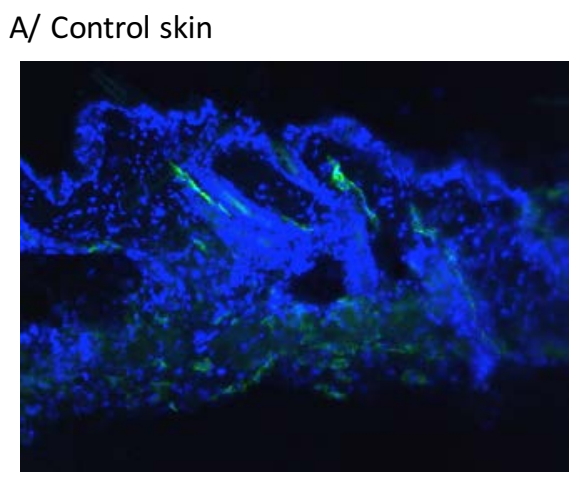

B/ Skin + B16 melanoma

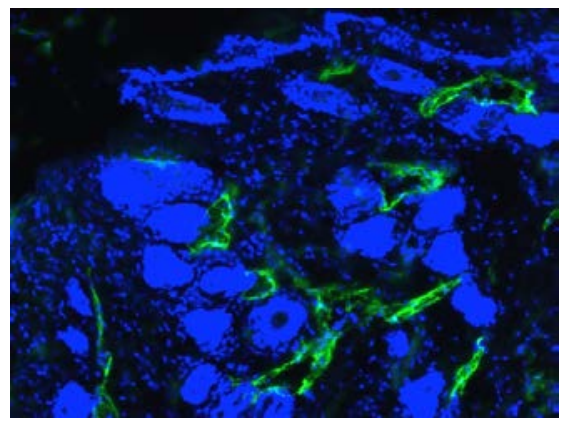

Fig. 4. Immunodetection of vessels (green) and cell nuclei (blue) on cryosections of control skin (A) and skin invaded by melanoma (B). 
Cells may be distinguished through their nucleus colored in blue, whereas the vascularization is noticeable with the green fluorophore. By comparing both normal skin and skin invaded by melanoma, one may observe an increase of vessels and vascular density in the presence of a tumor. This observation tends to confirm the interest of exploit the microwave range for melanoma detection, as such electromagnetic waves are very sensitive to water content. The relaxation frequency of water is indeed of the order of $20 \mathrm{GHz}$ at room temperature.

\section{B. Microwave sensor and portable test setup}

Fig. 5.A presents a schematic view of the corresponding test setup, which includes a sensor placed in contact with the skin, and connected on the other side to a Vector Network Analyzer with an RF cable. The characterization is realized with reflectometry. Data (reflection coefficient S11) are then treated to discriminate the detected skin based on dielectric properties in the microwave range.

To assure measurements in sterile environment (environment appropriate for biological studies on mice and rats), a portable VNA has been used for characterization up to $6 \mathrm{GHz}$. All equipment had also to be processed to avoid any contamination. Fig. 5.B presents a photography of experiment performed on unshaved sleeping mouse, whereas the right photography includes a view of the microstrip sensor on a shaved mouse.

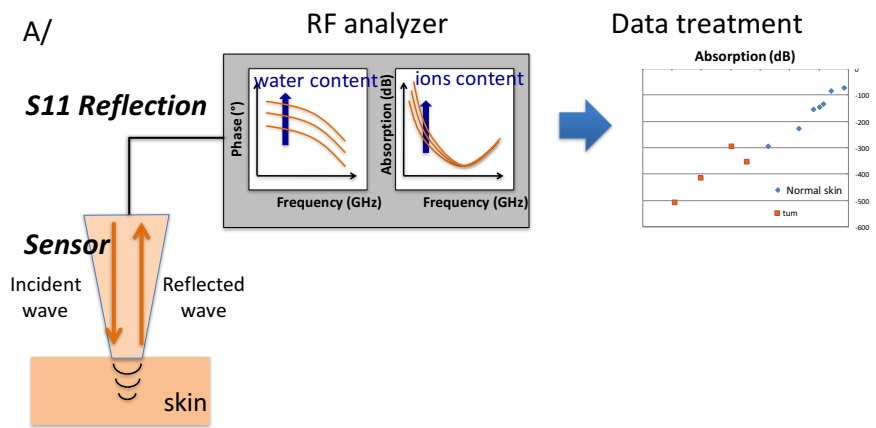

B/

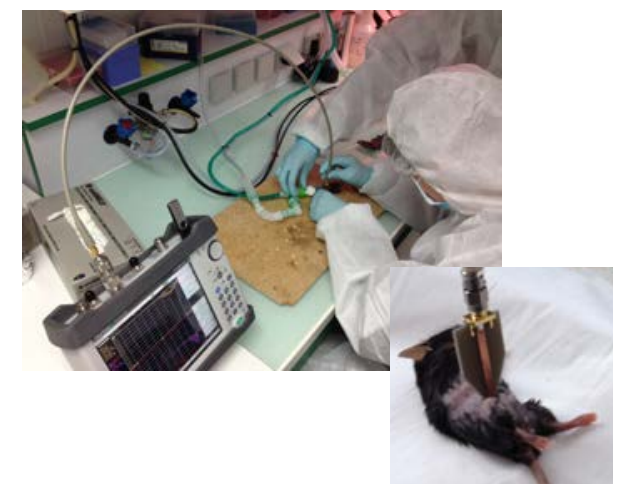

Fig. 5. Schematic view (A) and photography (B) of the portable microwave test setup for skin characterization of mouse in sterile environment.

The sensor corresponds to a microstrip line with a conductor width of $3 \mathrm{~mm}$ on a FR4 substrate of $1.6 \mathrm{~mm}$ thick. The advantages of the chosen technology are its low cost, its easy fabrication and the easy engineering of electromagnetic wave penetration through the thickness of dielectric and the width of the conductor. Such a topology is compatible with future miniaturization.

\section{Microwave results}

Two mice presenting a subcutaneous melanoma have been characterized in terms of reflection coefficient. To enhance a possible discrimination between skin invaded with melanoma and normal skin, the parameters have been integrated from 0 to $1.5 \mathrm{GHz}$ for both absorption and phase. The results are indicated in Fig. 6 for only one mouse. Similar results have been obtained for the second mouse.

Two distinct clouds of points can be noticed. The ones related to the skin invaded with melanoma exhibit larger values of absorption and phase compare to those corresponding to normal skin. This is in good agreement with the previous biological observation of increased vascularization within the tumor area. One explanation may be given by a larger amount of water in the tumorous tissue.

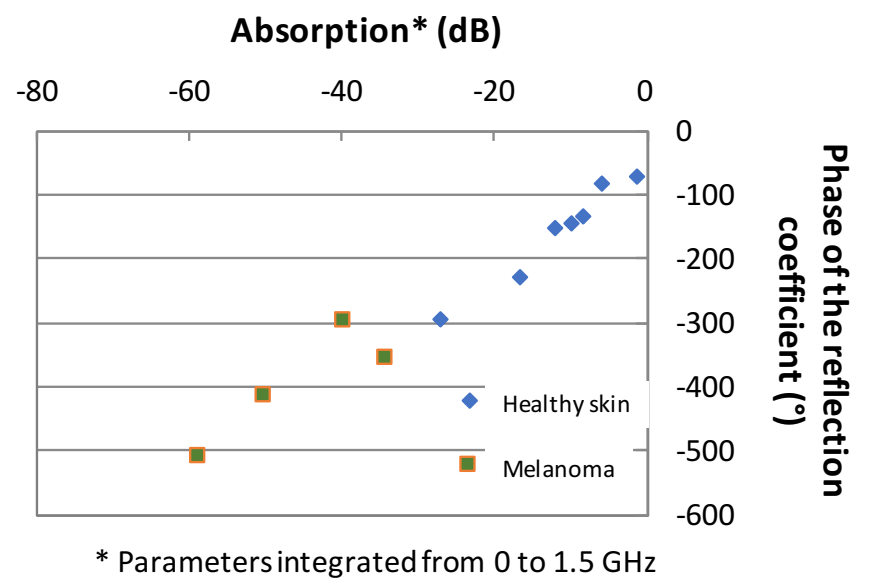

Fig. 6. Integrated absorption versus phase of the reflection coefficient depending of the skin type.

\section{CONCLUSIONS}

The preliminary results obtained both in vitro and in vivo on control skin and skin invaded with melanoma are encouraging. They confirm a possible discrimination of the tumorous skin compare to normal one. In vitro microwave measurements lead to higher dielectric contrasts in the case of the tumorous tissue, whereas in vivo ones also demonstrate enlarged parameters, which are coherent with the increased vascularization density and thus water quantity.

The results of this feasibility study therefore constitute an important basis for further investigations, which should now be performed to get statistical results, in complementarity with the miniaturization of the sensor to allow smaller spatial resolutions. 


\section{ACKNOWLEDGMENT}

This work was supported in part by the French INCa and Canceropole GSO and in part by LAAS-CNRS micro and nano technologies platform, member of the French RENATECH network.

\section{REFERENCES}

[1] M. Lazebnik, D. Popovic, L. McCartney, C. B. Watkins, M. J. Lindstrom, J. Harter, S. Sewall, T. Ogilvie, A.Magliocco, T.M. Breslin, W. Temple, D. Mew, J. H. Booske,M.Okoniewski, and S.C.Hagness, "A large-scale study of the ultrawideband microwave dielectric properties of normal, benign and malignant breast tissues obtained from cancer surgeries," Phys. Med. Biol., vol. 52, pp. 6093-6115, 2007.

[2] E. C. Fear, P. M. Meaney, and M. A. Stuchly, "Microwave for breast cancer detection?," IEEE Potentials, vol. 22, no. 1, pp. 12-18, Feb/Mar. 2003.

[3] Paul M. Meaney, Margaret W. Fanning, Roberta M. di FlorioAlexander, Peter A. Kaufman, Shireen, D. Geimer, Tian Zhou, Keith D. Paulsen, "Microwave tomography in the context of complex breast cancer imaging," IEEE EMBS conference, 2010, pp. 3398-3401.
[4] J P Grantis, R N Clarke, G T Symm and N M Spyrou, "In vivo dielectric properties of human skin from $50 \mathrm{MHz}$ to $2.0 \mathrm{GHz}$," Phys. Med. Biol., 1988, Vol. 33, No 5, 607-612.

[5] P. Mehta, K. Chand, D. Narayanswamy, D. G. Beetner, R. Zoughi, and W. V. Stoecker, "Microwave reflectometry as a novel diagnostic tool for detection of skin cancers," IEEE Trans. Instrum. Meas., vol. 55, no. 4, pp. 1309-1316, Aug. 2006.

[6] F. Töpfer, L. Emtestam, J. Oberhammer, "Dermatological verification of micromachined millimeter-wave skin cancer probe," IEEE IMS, 2014.

[7] F. Töpfer, J. Oberhammer, "Millimeter-Wave Tissue Diagnostics," IEEE Microwave Magazine, May 2015, pp. 97-113.

[8] P. Aberg, I. Nicander, S. Ollmar, "Minimally invasive electrical impedance spectroscopy of skin exemplified by skin cancer assessments », IEEE EMBC 2003, pp. 3211-3214.

[9] K. Grenier and D. Dubuc et al., "Integrated broadband microwave and microfluidic sensor dedicated to bioengineering," IEEE Trans.Microw. Theory Techn., vol. 57, no. 12, pp. 3246-3253, Dec. 2009.

[10] K. Grenier, D. Dubuc, T. Chen, F. Artis, T. Chretiennot, M. Poupot, and J-J. Fournié, "Recent Advances in Microwave-based Dielectric Spectroscopy at the Cellular Level for Cancer Investigations," IEEE Microwave Theory Tech., vol. 61, no. 5, pt. 2, pp. 2023-2030, 2013.

[11] F. Artis, T. Chen, T. Chrétiennot, J-J. Fournié, M. Poupot, D. Dubuc, K. Grenier, "Microwaving Biological Cells," IEEE Microwave Magazine, May 2015, pp. 87-96 